\title{
OFDM Synthetic Aperture Radar Imaging With Sufficient Cyclic Prefix
}

\author{
Tianxian Zhang and Xiang-Gen Xia, Fellow, IEEE
}

\begin{abstract}
The existing linear-frequency-modulated (or step frequency) and random noise synthetic aperture radar (SAR) systems may correspond to the frequency-hopping and direct-sequence spread spectrum systems in the past second- and third-generation wireless communications. Similar to the current and future wireless communications generations, in this paper, we propose the orthogonal frequency-division multiplexing (OFDM) SAR imaging, where a sufficient cyclic prefix (CP) is added to each OFDM pulse. The sufficient CP insertion converts an intersymbol interference (ISI) channel from multipaths into multiple ISI-free subchannels as the key in a wireless communications system, and analogously, it provides an inter-range-cell interference (IRCI)-free (high range resolution) SAR image in a SAR system. The sufficient $C P$ insertion along with our newly proposed SAR imaging algorithm, particularly for the OFDM signals, also differentiates this paper from all the existing studies in the literature on OFDM radar signal processing. Simulation results are presented to illustrate the high-range-resolution performance of our proposed CP-based OFDM SAR imaging algorithm.
\end{abstract}

Index Terms-Cyclic prefix (CP), inter-range-cell interference (IRCI), orthogonal frequency-division multiplexing (OFDM), swath width matched pulse (SWMP), synthetic aperture radar (SAR) imaging, zero sidelobes.

\section{INTRODUCTION}

$\mathbf{S}$ YNTHETIC aperture radar (SAR) can perform imaging well under almost all weather conditions [1], which, in the past decades, has received considerable attention. Several types of SAR systems using different transmitted signals have been well developed and analyzed, such as the linear frequency modulated (LFM) chirp radar [2], linear/random step frequency radar [1], [3], and random noise radar [4]-[6].

Recently, orthogonal frequency-division multiplexing (OFDM) signals have been used in radar applications, which may provide opportunities to achieve ultrawideband radar. OFDM radar signal processing was first presented in [7] and

Manuscript received September 17, 2013; revised December 24, 2013 and March 18, 2014; accepted May 7, 2014. The work of T. Zhang was supported in part by the Fundamental Research Funds for the Central Universities under Grant ZYGX2012YB008 and in part by the China Scholarship Council and was done when he was visiting the University of Delaware, Newark, DE 19716, USA. The work of X.-G. Xia was supported in part by the Air Force Office of Scientific Research under Grant FA9550-12-1-0055.

T. Zhang is with the School of Electronic Engineering, University of Electronic Science and Technology of China, Chengdu 611731, China (e-mail: tianxian.zhang@gmail.com).

X.-G. Xia is with the Department of Electrical and Computer Engineering, University of Delaware, Newark, DE 19716, USA (e-mail: xxia@ee.udel.edu).

Color versions of one or more of the figures in this paper are available online at http://ieeexplore.ieee.org.

Digital Object Identifier 10.1109/TGRS.2014.2322813 was also studied in [8]-[13]. Adaptive OFDM radar was investigated for moving target detection and low-grazing angle target tracking in [14]-[16]. Using OFDM signals for SAR applications was proposed in [17]-[23]. In [17]-[19], adaptive OFDM signal design was studied for range ambiguity suppression in SAR imaging. The reconstruction of the cross-range profiles is studied in [22] and [23]. The signal processing of a passive OFDM radar using digital audio broadcast, digital video broadcast, Wireless Fidelity (Wi-Fi) or worldwide inoperability for microwave access (WiMAX) signals for target detection and SAR imaging was investigated in [24]-[30]. However, all the existing OFDM radar (including SAR) signal processing is on radar waveform designs with ambiguity function analyses to mitigate the interferences between range/cross-range cells using multicarrier signals similar to the conventional waveform designs, and the radar receivers, such as SAR imaging algorithms, are basically not changed. The most important feature of OFDM signals in communications systems, namely, converting an intersymbol interference (ISI) channel to multiple ISI-free subchannels, when a sufficient cyclic prefix $(\mathrm{CP})$ is inserted, has not been utilized so far in the literature. In this paper, we will fully take this feature of the OFDM signals into account to propose OFDM SAR imaging, where a sufficient $\mathrm{CP}$ is added to each OFDM pulse, as the next-generation high-range-resolution SAR imaging. In our proposed SAR imaging algorithm, not only the transmission side but also the receive side is different from the existing SAR imaging methods. To further explain it, let us briefly overview some of the key signalings in SAR imaging.

To achieve long distance imaging, a pulse with long enough time duration is used to carry enough transmit energy [31]. The received pulses from different scatterers are overlapped with each other and cause energy interferences between these scatterers. To mitigate the impact of the energy interferences and achieve high resolution, the transmitted pulse is coded using frequency or phase modulation (i.e., LFM signal and step frequency signal) or random noise type signals in random noise radar to achieve a bandwidth $B$ which is large compared to that of an uncoded pulse with the same time duration [31]. This is similar to the spread spectrum technique in communications systems. Then, pulse compression techniques are applied at the receiver to yield a narrow compressed pulse response. Thus, the reflected energies from different range cells can be distinguished [31]. However, the energy interferences between different range cells, which we regard as inter-range-cell interference (IRCI), still exist because of the sidelobes of the ambiguity function of the transmitted signal, whose sidelobe 
magnitude is roughly $\sqrt{N}$ if the main lobe of the modulated signal pulse is $N$ [32]. This IRCI is much significant in SAR imaging [2]. Despite of the IRCI, the existing well-known/used SAR are LFM (or step frequency) SAR [2], [3] and random noise SAR [4]-[6].

We are adopting the OFDM technique [33] that is the key technology in the latest wireless communications standards, such as long-term evolution (LTE) [34] and Wi-Fi. If we think about only one user in a wireless communications system, there are similarity and difference between wireless communications systems and SAR imaging systems. The similarity is that both systems are transmitting and receiving signals reflected from various scatterers and the numbers of multipaths depend on the transmitted signal bandwidths. The difference is that, in communications systems, the receiver cares about the transmitted signal, while in SAR imaging systems, the receiver cares about the scatterers within different range cells (with different time delays) that reflect and cause multipath signals. The multipaths cause ISI in communications, while the multipaths cause the IRCI in SAR imaging systems. The higher the bandwidth is, the more multipaths there are in communications systems, and the more range cells there are for a fixed imaging scene (or swath width), i.e., the higher the range resolution is, in SAR imaging systems. The existing well-known/used LFM (or step frequency) and random noise SAR systems, in fact, correspond to the two spread spectrum systems, i.e., frequency hopping and direct sequence in CDMA systems [35], which work well in nonhigh-bandwidth wireless communications systems, such as the second and third generations of cellular communications, of less than 10-MHz (roughly) bandwidth. They, however, may not work well for a system of a much higher signal bandwidth, such as $20 \mathrm{MHz}$ in LTE, due to the severe ISI caused by too many multipaths. In contrast, since OFDM with a sufficient CP can convert an ISI channel to multiple ISI-free subchannels, as mentioned earlier, it is used in the latest LTE and Wi-Fi standards. As an analogy, one expects that LFM and random noise SAR may not work too well for high-bandwidth radar systems where there are too many range cells in one cross range, which cause severe IRCI due to the significant sidelobes of the ambiguity functions of the LFM and random signals. On the other hand, in order to have a high range resolution, a high bandwidth is necessary. Therefore, borrowing from wireless communications, in this paper, we propose to use OFDM signals with sufficient $\mathrm{CP}$ to deal with the IRCI problem as a next-generation SAR imaging to produce a high-rangeresolution SAR image. We show that, in our proposed OFDM SAR imaging with a sufficient $\mathrm{CP}$, the sidelobes are ideally zero, and for any range cell, there will be no IRCI from other range cells in one cross range.

Another difference for OFDM communications systems and CP-based OFDM SAR systems is as follows. It is known in communications that, for an OFDM system, a Doppler frequency shift is not desired, while the azimuth domain (or cross-range direction) in a SAR imaging system is, however, generated from the relative Doppler frequency shifts between the radar platform and the scatterers. One might ask how the OFDM signals are used to form a SAR image. This question is not difficult to answer. The range distance between the radar

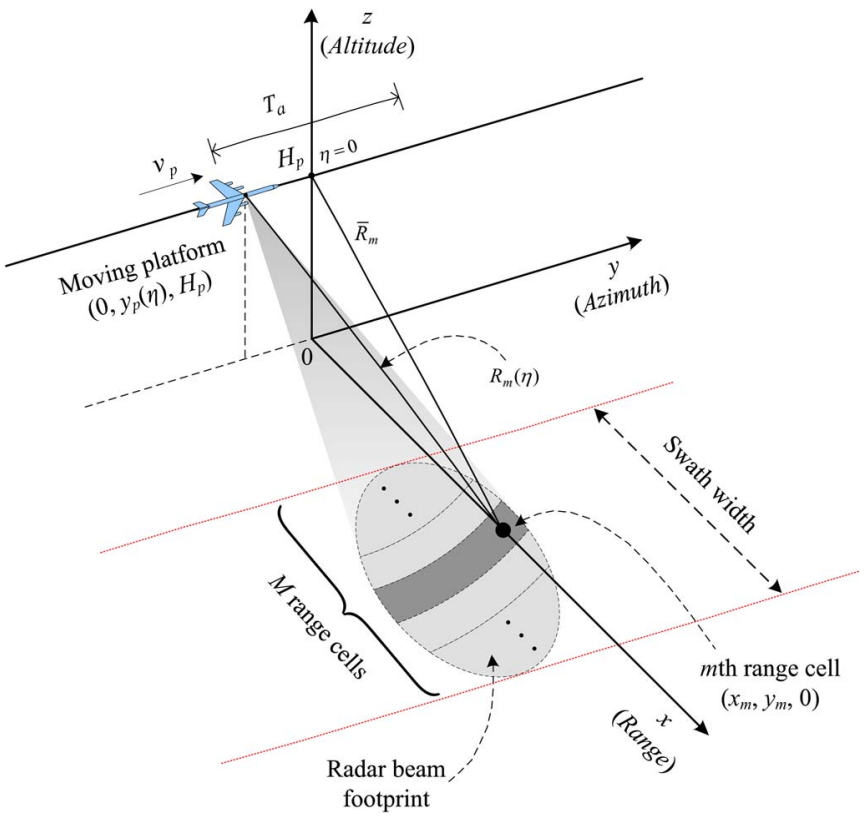

Fig. 1. Monostatic stripmap SAR geometry.

platform and image scene is known, and the radar platform moving velocity is known too. Thus, the Doppler shifts are also known, which can be used to generate the cross ranges similar to other SAR imaging techniques and can be also used to compensate the Doppler shift inside one cross range and correct the range migration.

This paper is organized as follows. In Section II, we propose our CP-based OFDM SAR imaging algorithm. In Section III, we present some simulations to illustrate the high-rangeresolution property of the proposed CP-based OFDM SAR imaging and also the necessity of a sufficient $\mathrm{CP}$ insertion in an OFDM signal. In Section IV, we conclude this paper and point out some future research problems.

\section{System Model AND CP-BASED OFDM SAR IMAGING}

In this section, we first describe the OFDM SAR signal model and then propose the corresponding SAR imaging algorithm.

\section{A. OFDM SAR Signal Model}

In this paper, we consider the monostatic broadside stripmap SAR geometry as shown in Fig. 1. The radar platform is moving parallelly to the $y$-axis with an instantaneous coordinate $\left(0, y_{p}(\eta), H_{p}\right), H_{p}$ is the altitude of the radar platform, $\eta$ is the relative azimuth time referenced to the time of zero Doppler, $T_{a}$ is the synthetic aperture time defined by the azimuth time extent that the target stays in the antenna beam. For convenience, let us choose the azimuth time origin $\eta=0$ to be the zero Doppler sample. Consider an OFDM signal with $N$ subcarriers and a bandwidth of $B \mathrm{~Hz}$, and let $\boldsymbol{S}=\left[S_{0}, S_{1}, \ldots, S_{N-1}\right]^{T}$ represent the complex weights transmitted over the subcarriers and $\sum_{k=0}^{N-1}\left|S_{k}\right|^{2}=N$. Then, a discrete time OFDM signal is 
the inverse fast Fourier transform (IFFT) of the vector $\boldsymbol{S}$, and the OFDM pulse is

$$
s(t)=\frac{1}{\sqrt{N}} \sum_{k=0}^{N-1} S_{k} \exp \{j 2 \pi k \Delta f t\}, \quad t \in\left[0, T+T_{G I}\right]
$$

where $\Delta f=B / N=1 / T$ is the subcarrier spacing. $\left[0, T_{G I}\right)$ is the time duration of the guard interval that corresponds to the $\mathrm{CP}$ in the discrete time domain as we shall see later in more details. Its length $T_{G I}$ will be specified later too, and $T$ is the length of the OFDM signal excluding CP. Due to the periodicity of the exponential function $\exp (\cdot)$ in (1), the tail part of $s(t)$ for $t$ in $\left(T, T+T_{G I}\right]$ is the same as the head part of $s(t)$ for $t$ in $\left[0, T_{G I}\right)$. Let $f_{c}$ be the carrier frequency of operation, and the transmitted signal is given by

$s_{1}(t)=\operatorname{Re}\left\{\frac{1}{\sqrt{N}} \sum_{k=0}^{N-1} S_{k} \exp \left\{j 2 \pi f_{k} t\right\}\right\}, \quad t \in\left[0, T+T_{G I}\right]$

where $f_{k}=f_{c}+k \Delta f$ is the $k$ th subcarrier frequency.

After the demodulation to baseband, the complex envelope of the received signal from a static point target in the $m$ th range cell can be written in terms of fast time $t$ and slow time $\eta$

$$
\begin{aligned}
u_{m}(t, \eta)= & g_{m} \varepsilon_{a}(\eta) \exp \left\{-j 4 \pi f_{c} \frac{R_{m}(\eta)}{c}\right\} \\
& \times \frac{1}{\sqrt{N}} \sum_{k=0}^{N-1} S_{k} \exp \left\{\frac{j 2 \pi k}{T}\left[t-\frac{2 R_{m}(\eta)}{c}\right]\right\} \\
& +w(t, \eta), t \in\left[\frac{2 R_{m}(\eta)}{c}, \frac{2 R_{m}(\eta)}{c}+T+T_{G I}\right]
\end{aligned}
$$

where $\varepsilon_{a}(\eta)=p_{a}^{2}(\theta(\eta))$ is the azimuth envelope, $p_{a}(\theta) \approx$ $\operatorname{sinc}\left(0.886 \theta / \beta_{b w}\right)$ [2], $\operatorname{sinc}(x)=\sin (x) / x$ is the sinc function, $\theta$ is the angle measured from the boresight in the slant range plane, $\beta_{b w}=0.866 \lambda / L_{a}$ is the azimuth beamwidth, $L_{a}$ is the effective length of the antenna, $g_{m}$ is the radar cross section (RCS) coefficient caused from the scatterers in the $m$ th range cell within the radar beam footprint, and $c$ is the speed of light. $w(t, \eta)$ represents the noise. $R_{m}(\eta)$ is the instantaneous slant range between the radar and the $m$ th range cell with the coordinate $\left(x_{m}, y_{m}, 0\right)$, and it can be written as

$$
R_{m}(\eta)=\sqrt{\bar{R}_{m}^{2}+\left(y_{m}-y_{p}(\eta)\right)^{2}}=\sqrt{\bar{R}_{m}^{2}+v_{p}^{2} \eta^{2}}
$$

where $\bar{R}_{m}=\sqrt{x_{m}^{2}+H_{p}^{2}}$ is the slant range when the radar platform and the target in the $m$ th range cell are the closest approach and $v_{p}$ is the effective velocity of the radar platform.

Then, the complex envelope of the received signal from all the range cells in a swath can be written as

$$
u(t, \eta)=\sum_{m} u_{m}(t, \eta)
$$

At the receiver, with the A/D converter, the received signal is sampled with sampling interval $T_{s}=1 / B$, and the range

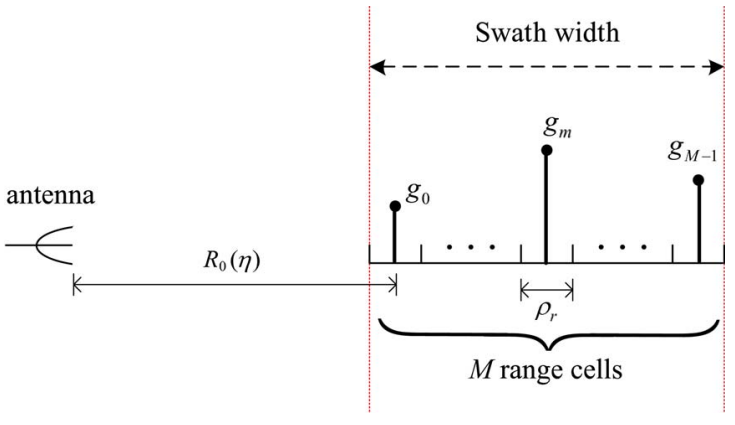

Fig. 2. Illustration diagram of one range line.

resolution is $\rho_{r}=c / 2 B$. Assume that the swath width for the radar is $R_{w}$. Let $M=R_{w} / \rho_{r}$ that is determined by the radar system. Then, a range profile can be divided into $M$ range cells as shown in Fig. 2. As we mentioned earlier, the main reason why OFDM has been successfully adopted in both recent wireline and wireless communications systems is its ability to deal with multipaths (they cause ISI in communications) that become more severe when the signal bandwidth is larger. In our radar applications here, the response of each range cell, formed by the summation of the responses of all scatterers within this range cell, contains its own delay and phase. Thus, to a transmitted pulse, each range cell can be regarded as one path of communications. $M$ range cells correspond to $M$ paths. Excluding one main path (i.e., the nearest range cell), there will be $M-1$ multipaths. To convert the ISI caused from the multipaths to the ISI-free case in communications, a guard interval (or CP) needs to be added to each OFDM block, and the $\mathrm{CP}$ length cannot be smaller than the number of multipaths that is $M-1$ in this paper. Although, in the radar application here, ISI is not the concern, the $M$ range cell paths are superposed (or interfered) together in the radar return signal, which is the same as the ISI in communications. Therefore, in order to convert these interfered $M$ range cells to individual range cells without any IRCI, similar to OFDM systems in communications, the $\mathrm{CP}$ length should be at least $M-1$. For convenience, we use CP length $M-1$ in this paper, i.e., a CP of length $M-1$ is added at the beginning of an OFDM pulse, and then, the guard interval length $T_{G I}$ in the analog transmission signal is $T_{G I}=(M-1) T_{s}$. Notice that $T=N T_{s}$, so the time duration of an OFDM pulse is $T_{o}=T+T_{G I}=(N+M-1) T_{s}$. In this paper, we assume $N \geq M$, i.e., the number of subcarriers of the OFDM signal is at least the number of range cells in a swath (or a cross range), which is similar to the application in communications [33], [35]. When $N<M$, the IRCI occurs, and the detailed reason will be seen later.

In communications applications, to achieve a high transmission throughput, the OFDM pulses are transmitted consecutively as shown in Fig. 3(a). However, in SAR imaging applications, for the monostatic case, the transmitter and receiver share the same antenna, which cannot both transmit and receive signals at the same time, and transmission throughput is not a concern. Thus, transmitted signals and radar return signals are usually separated in time. This implies that a reasonable receive window is needed between two consecutive pulses as shown in Fig. 3(b). For convenience, similar to what is 

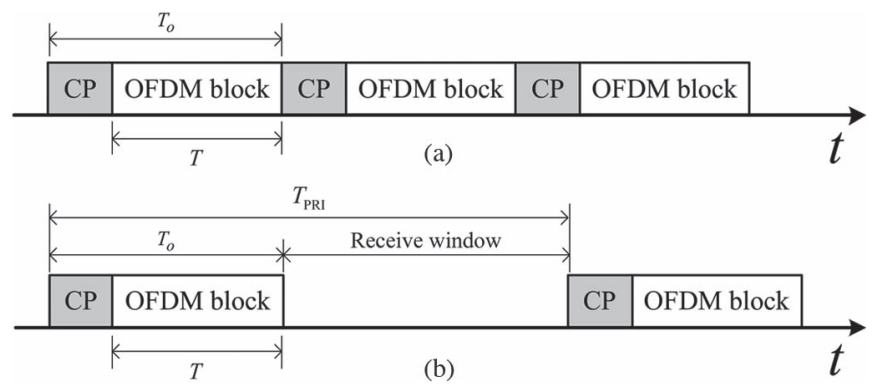

Fig. 3. Transmission comparison of OFDM signals in (a) communications systems and (b) SAR systems.

commonly done in SAR systems, in this paper, we assume that the pulse repetition interval (PRI) is long enough so that all of the range cells in a swath fall within the receive window. Therefore, the PRI length $T_{\mathrm{PRI}}$ should be

$$
T_{\mathrm{PRI}}=\frac{1}{\mathrm{PRF}}>\left(\frac{2 R_{w}}{c}+T_{o}\right)
$$

where $R_{w}=M \rho_{r}$ is the swath width. We want to emphasize here that, in our common SAR imaging applications, the pulse repetition frequency (PRF) may not be too high [2] and there is sufficient time duration to add a CP (a guard interval) for an OFDM pulse.

For Fig. 2, we notice that $R_{m}(\eta)=R_{0}(\eta)+m \rho_{r}$. Thus, $t-$ $\left(2 R_{m}(\eta) / c\right)$ in (3) can be written as

$$
\begin{aligned}
t-\frac{2 R_{m}(\eta)}{c} & =t-\frac{2\left(R_{0}(\eta)+m \rho_{r}\right)}{c} \\
& =t-t_{0}(\eta)-m T_{s}
\end{aligned}
$$

where, for each $\eta$, the constant time delay $t_{0}(\eta)=2 R_{0}(\eta) / c$ is independent of $m$. Let the sampling be aligned with the start of the received pulse after $t_{0}(\eta)$ seconds for the first arriving version of the transmitted pulse. Combining with (3), (7), and (5), $u(t, \eta)$ can be converted to the discrete time linear convolution of the transmitted sequence with the weighting RCS coefficients $d_{m}$, and the received sequence can be written as

$$
u_{i}=\sum_{m=0}^{M-1} d_{m} s_{i-m}+w_{i}, \quad i=0,1, \ldots, N+2 M-3
$$

where

$$
d_{m}=g_{m} \varepsilon_{a}(\eta) \exp \left\{-j 4 \pi f_{c} \frac{R_{m}(\eta)}{c}\right\}
$$

in which $4 \pi f_{c}\left(R_{m}(\eta) / c\right)$ in the exponential is the azimuth phase and $s_{i}$ is the complex envelope of the OFDM pulse in (1) with time duration $t \in\left[0, T+T_{G I}\right]$ for $T=N T_{s}$ and $T_{G I}=(M-1) T_{s}$. After sampling at $t=i T_{s}$, (1) can be recast as

$$
\begin{aligned}
s_{i}=s\left(i T_{s}\right)=\frac{1}{\sqrt{N}} \sum_{k=0}^{N-1} S_{k} \exp \left\{\frac{j 2 \pi k i}{N}\right\}, & \\
i & =0,1, \ldots, N+M-2,
\end{aligned}
$$

and $s_{i}=0$ if $i<0$ or $i>N+M-2$.
Notice that the transmitted sequence with $\mathrm{CP}$ is $\tilde{\boldsymbol{s}}=$ $\left[s_{0}, s_{1}, \ldots, s_{N+M-2}\right]^{T}$, where $\left[s_{0}, \ldots, s_{M-2}\right]^{T}=\left[s_{N}, \ldots\right.$, $\left.s_{N+M-2}\right]^{T}$. The vector $s=\left[s_{0}, s_{1}, \ldots, s_{N-1}\right]^{T}$ is indeed the IFFT of the vector $\boldsymbol{S}=\left[S_{0}, S_{1}, \ldots, S_{N-1}\right]^{T}$.

\section{B. Range Compression}

When the signal in (8) is received, the first and last $M-1$ samples ${ }^{1}$ are removed, and then, we obtain

$u_{n}=\sum_{m=0}^{M-1} d_{m} s_{n-m}+w_{n}, \quad n=M-1, M, \ldots, N+M-2$.

Then, the received signal $\boldsymbol{u}=\left[u_{M-1}, u_{M}, \ldots, u_{N+M-2}\right]^{T}$ is

$\left[\begin{array}{c}u_{M-1} \\ u_{M} \\ \vdots \\ u_{N+M-2}\end{array}\right]$

$$
=\left[\begin{array}{cccccccc}
d_{M-1} & \cdots & d_{0} & \cdots & \cdots & 0 & \cdots & 0 \\
0 & d_{M-1} & \cdots & d_{0} & \cdots & 0 & \cdots & 0 \\
\vdots & \ddots & \ddots & \ddots & \ddots & \ddots & \ddots & \vdots \\
0 & \cdots & 0 & \cdots & \cdots & d_{M-1} & \cdots & d_{0}
\end{array}\right]
$$

$$
\times\left[\begin{array}{c}
s_{0} \\
\vdots \\
s_{M-1} \\
s_{M} \\
\vdots \\
s_{N+M-2}
\end{array}\right]+\left[\begin{array}{c}
w_{M-1} \\
w_{M} \\
\vdots \\
w_{N+M-2}
\end{array}\right] .
$$

Since $\left[s_{0}, \ldots, s_{M-2}\right]^{T}=\left[s_{N}, \ldots, s_{N+M-2}\right]^{T}$, it is not hard to see that the vector $\tilde{s}=\left[s_{0}, s_{1}, \ldots, s_{N+M-2}\right]^{T}$ in (12) can be replaced by its tail part, ${ }^{2} s^{\prime}$ which can be also seen in, for example, [35, Ch. 12.4], and then, the matrix representation (12) is equivalent to the following representation:

$$
\boldsymbol{u}=\boldsymbol{H} \boldsymbol{s}^{\prime}+\boldsymbol{w}
$$

where $s^{\prime}=\left[s_{M-1}, s_{M}, \ldots, s_{N+M-2}\right]^{T}=\left[s_{M-1}, \ldots, s_{N-1}, s_{0}\right.$, $\left.\ldots, s_{M-2}\right]^{T}, \boldsymbol{w}=\left[w_{M-1}, w_{M}, \ldots, w_{N+M-2}\right]^{T}$, and $\boldsymbol{H}$ is built by superposing the first $M-1$ columns of the weighting RCS coefficient matrix in (12) to its last $M-1$ columns.

\footnotetext{
${ }^{1}$ The reason to remove both the head and tail $M-1$ samples is because the total number of received signal samples in $(8)$ is $N+2(M-1)$. Because of the receive window between the OFDM pulses as shown in Fig. 3(b), the tail $M-1$ samples in (8) are not affected by the follow-up OFDM pulses. However, they do not have the full $M$ RCS coefficients from all the $M$ range cells. If there is no receive window between the OFDM pulses, the transmission is shown in Fig. 3(a) as in communications, and we only remove the head $M-$ 1 samples from the received signal sequence $u_{n}$ and use the next $N$ samples of $u_{n}$ starting from $n=M-1$.

${ }^{2}$ This part is slightly different from what appears in communications applications (see [33, Ch. 5.2]) where the vector $\boldsymbol{s}^{\prime}$ in (13) is replaced by the head part, $\boldsymbol{s}$, of the vector $\tilde{\boldsymbol{s}}$.
} 
Moreover, $\boldsymbol{H}$ can be given by the following $N$ by $N$ matrix:

$$
\boldsymbol{H}=\left[\begin{array}{ccccccc}
d_{0} & 0 & \cdots & 0 & d_{M-1} & \cdots & d_{1} \\
\vdots & \ddots & \ddots & \vdots & \ddots & \ddots & \vdots \\
d_{M-2} & \cdots & d_{0} & 0 & \cdots & 0 & d_{M-1} \\
d_{M-1} & d_{M-2} & \cdots & d_{0} & 0 & \cdots & 0 \\
0 & \ddots & \ddots & \vdots & \ddots & \ddots & \vdots \\
\vdots & \ddots & d_{M-1} & d_{M-2} & \cdots & d_{0} & 0 \\
0 & \cdots & 0 & d_{M-1} & d_{M-2} & \cdots & d_{0}
\end{array}\right] .
$$

One can see that the matrix $\boldsymbol{H}$ in (14) is a circulant matrix that can be diagonalized by the discrete Fourier transform (DFT) matrix of the same size.

The OFDM demodulator then performs a fast Fourier transform (FFT) on the vector $\boldsymbol{u}$

$U_{k}=\frac{1}{\sqrt{N}} \sum_{n=0}^{N-1} u_{n+M-1} \exp \left\{\frac{-j 2 \pi k n}{N}\right\}, k=0,1, \ldots, N-1$.

From (13) and (14), the aforementioned $U_{k}$ can be expressed as

$$
U_{k}=D_{k} S_{k}^{\prime}+W_{k}, \quad k=0,1, \ldots, N-1
$$

where $\left[S_{0}^{\prime}, S_{1}^{\prime}, \ldots, S_{N-1}^{\prime}\right]^{T}$ is the FFT of the vector $\boldsymbol{s}^{\prime}$, a cyclic shift of the vector $s$ of amount $M-1$, i.e., $S_{k}^{\prime}=$ $S_{k} \exp \{j 2 \pi k(M-1) / N\}, W_{k}$ is the FFT of the noise, and

$$
D_{k}=\sum_{m=0}^{M-1} d_{m} \exp \left\{\frac{-j 2 \pi m k}{N}\right\} \text {. }
$$

Then, the estimate of $D_{k}$ is

$$
\begin{aligned}
\hat{D}_{k} & =\frac{U_{k}}{S_{k}^{\prime}}=\frac{U_{k}}{S_{k} \exp \left\{\frac{j 2 \pi k(M-1)}{N}\right\}} \\
& =D_{k}+\frac{W_{k}}{S_{k}} \exp \left\{\frac{-j 2 \pi k(M-1)}{N}\right\}, k=0,1, \ldots, N-1 .
\end{aligned}
$$

Notice that, if $S_{k}$ is small, the noise is enhanced. Thus, for the constraint condition $\sum_{k=0}^{N-1}\left|S_{k}\right|^{2}=N$, from (18), the optimal signal $S_{k}$ should have a constant module for all $k$ 's.

The vector $\boldsymbol{D}=\left[D_{0}, D_{1}, \ldots, D_{N-1}\right]^{T}$ is indeed the $N$ point FFT of $\sqrt{N} \gamma$, where $\gamma$ is the weighting RCS coefficient vector

$$
\gamma=[d_{0}, d_{1}, \ldots, d_{M-1}, \underbrace{0, \ldots, 0}_{N-M}]^{T} .
$$

Therefore, the estimate of $d_{m}$ can be achieved by the $N$-point IFFT on the vector $\hat{\boldsymbol{D}}=\left[\hat{D}_{0}, \hat{D}_{1}, \ldots, \hat{D}_{N-1}\right]^{T}$

$$
\hat{d}_{m}=\frac{1}{\sqrt{N}} \sum_{k=0}^{N-1} \hat{D}_{k} \exp \left\{\frac{j 2 \pi m k}{N}\right\}, \quad m=0, \ldots, M-1 .
$$

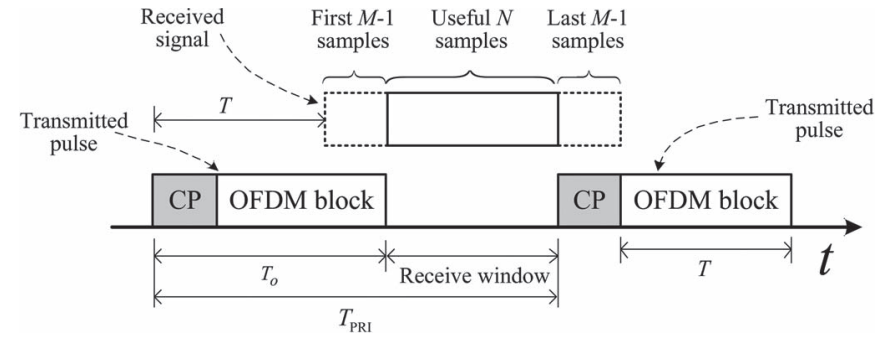

Fig. 4. Transmitted and received timing of OFDM SAR system for $M=N$.

Then, we obtain the following estimates of the $M$ range cell weighting RCS coefficients:

$$
\hat{d}_{m}=\sqrt{N} d_{m}+\tilde{w}_{m}^{\prime}, \quad m=0, \ldots, M-1
$$

where $\tilde{w}_{m}^{\prime}$ is from the noise and its variance is the same as that in (18) since the IFFT implemented in (20) is a unitary transform. In (21), $d_{m}$ can be completely recovered without any IRCI from other range cells. From (9), when $d_{m}$ is determined, the RCS coefficients $g_{m}$ are determined and vice versa.

For $N>M$, there are some zeros in the vector $\gamma$ in (19). Considering (19) and (20), we notice that part of the transmitted OFDM sequence is used to estimate the unreal weighting RCS coefficients, i.e., the zeros in $\gamma$.

When $N=M$, there are no zeros included in the vector $\gamma$ in (19). We name this special case as swath width matched pulse (SWMP). The OFDM pulse length (excluding CP) $T=N T_{s}$ and the swath width $M \rho_{r}$ follow the relationship of $M \rho_{r}=$ $c T / 2$, i.e., the range resolution of a pulse with time duration $T$ is just the swath width $M \rho_{r}$ without range compression processing. Moreover, $c T / 2$ is the maximal swath width that we can obtain without IRCI. Thus, the optimal time duration of the OFDM pulse is $T_{o}=(2 N-1) T_{s}$ with CP length $N-$ 1 , which is the maximal possible CP length for an OFDM sequence of length $N$.

Since the first and last $M-1$ samples of the received sequence $u_{i}$ in (8) are removed, the receiver only needs to sample the received signal from $t=T_{0}$ to $t=T_{0}+T$, even when the radar return signal starts to arrive at time $t=T$ as shown in Fig. 4. Therefore, the minimum range of the OFDM radar is $c T / 2$, the same as that of the traditional pulse radar of transmitted pulse length $T$. Notice that the minimum range is just the same as the maximal swath width. Thus, if we want to increase the swath width to, e.g., $10 \mathrm{~km}$, the transmitted pulse duration $T_{o}$ should be increased to about $133.3 \mu \mathrm{s}$, and the minimum range is increased ${ }^{3}$ to $10 \mathrm{~km}$. Also, the minimum receive window is just the OFDM pulse length $T$ (excluding $\mathrm{CP}$ ) as shown in Fig. 4, and the PRI length follows $T_{\mathrm{PRI}}>T_{o}+T$. As a remark, different from the applications in communications where the $\mathrm{CP}$ is an overhead and may reduce the transmission

\footnotetext{
${ }^{3}$ The pulse length here is much longer than the traditional radar pulse, and the number $N$ of subcarriers in OFDM signals is large too. While long pulses of LFM signals need high frequency linearity and stability and thus are not easy to generate, although long LFM pulses are not necessary, long OFDM pulses (i.e., large $N$ ) are not difficult to generate since OFDM signals can be easily generated by the IFFT operation as in (1). Moreover, the conventional multiple channel SAR technology [36] for wide imaged swath width can be used to reduce the OFDM pulse length and the minimum range.
} 
data rate, in the SWMP case in SAR applications here, the longer the CP length is, the less the IRCI is, which leads to a better (high resolution) SAR image.

When $N<M$, according to (10), the signal vector $\left[s_{0}, \ldots\right.$, $\left.s_{M-1}, s_{M}, \ldots, s_{N+M-2}\right]^{T}$ in (12) is $\left[s_{0}, \ldots, s_{N-1}, s_{0}\right.$, $\left.\ldots, s_{N-1}, \ldots, s_{(N+M-2)_{N}}\right]^{T}$, where $(n)_{N}$ is the residue of $n$ modulo $N$. Thus, the $N$ by $N$ matrix $\boldsymbol{H}$ in (13) and (14) becomes

$$
\boldsymbol{H}=\left[\begin{array}{cccc}
\tilde{d}_{0} & \tilde{d}_{N-1} & \cdots & \tilde{d}_{1} \\
\tilde{d}_{1} & \tilde{d}_{0} & \cdots & \tilde{d}_{2} \\
\vdots & \vdots & \ddots & \vdots \\
\tilde{d}_{N-1} & \tilde{d}_{N-2} & \cdots & \tilde{\tilde{d}}_{0}
\end{array}\right]
$$

where $\tilde{d}_{n}=\sum_{i: 0 \leq i N+n \leq M-1} d_{i N+n}, n=0, \ldots, N-1$. One can see that $\tilde{d}_{n}$ is a summation of weighting RCS coefficients from several range cells, i.e., each $\tilde{d}_{n}$ has IRCI. Then, following the OFDM approach (15)-(21), what we can solve is the superposed weighting RCS coefficients $\tilde{d}_{n}$, i.e., IRCI occurs. This is the reason why we require $N \geq M$ in this paper.

After the range compression, combining (3), (8), (9), and (21), the range-compressed signal can be written as

$$
\begin{aligned}
u_{r a}(t, \eta)=\sqrt{N} & \sum_{m=0}^{M-1} \hat{g}_{m} \delta\left(t-\frac{2 R_{m}(\eta)}{c}\right) \varepsilon_{a}(\eta) \\
& \times \exp \left\{-j 4 \pi f_{c} \frac{R_{m}(\eta)}{c}\right\}+w_{r a}(t, \eta),
\end{aligned}
$$

where $\delta\left(t-\left(2 R_{m}(\eta) / c\right)\right)$ is the delta function with a nonzero value at $t=2 R_{m}(\eta) / c$, which indicates that the estimates $\hat{g}_{m}$ of the RCS coefficient values $g_{m}$ are not affected by any IRCI from other range cells after the range compression. $\hat{g}_{m}$ can be obtained via (9) using the estimate $\hat{d}_{m}$ in (20). In the delta function, the target range migration is incorporated via the azimuth varying parameter $2 R_{m}(\eta) / c$. Also, the azimuth phase in the exponential is unaffected by the range compression.

Comparing with (3), we notice that the range compression gain in (23) is equal to $\sqrt{N}$, and the noise powers are the same in (23) and (3) when $S_{k}$ have constant module. Thus, the signal-to-noise ratio (SNR) gain after the range compression is $N$. For an LFM signal pulse with time duration $T_{L}=T_{o}=$ $(N+M-1) T_{s}$ and the same transmitted signal energy as in (1), it is well known that the SNR gain after range compression is $N+M-1$, which is equal to the time-bandwidth product of the LFM signal pulse [2]. Clearly, $N<N+M-1<2 N$. This implies that the LFM range compression SNR gain is larger (but not too much larger) than that of the OFDM pulse. However, the IRCI exists because of the sidelobes of the ambiguity function, resulting in a significant imaging performance degradation. In fact, the sidelobe magnitude is roughly on the order of $\sqrt{N}$ in this case, and all the sidelobes from scatterers in all other $M-1$ range cells will be added to the $m$ th range cell for an arbitrary $m, 0 \leq m \leq M-1$. One can see that, when $M$ is roughly more than $\sqrt{N}$, the scatterers in the $m$ th range cell will be possibly buried by the sidelobes of the scatterers in other range cells and therefore cannot be well detected and imaged. This is similarly true for a random noise radar. In contrast, since the sidelobes are ideally 0 in the OFDM signal here, all $M$ scatterers can be ideally detected and imaged without any IRCI as long as $N \geq M$, which may provide a high-range-resolution image.

\section{Discussion on the Design of Weights $S_{k}$}

As one has seen from (15)-(21), in order to estimate the weighting RCS coefficients, the noise needs to be divided by $S_{k}$, which may be significantly enhanced if $S_{k}$ is small. As mentioned earlier, in this regard, the optimal weights $S_{k}$ should have a constant module.

A special case of constant modular weights is that all $S_{k}$ are the same, i.e., a constant. In this special case, the signal sequence $s_{i}$ to transmit is the delta sequence, i.e., $s_{0}=\sqrt{N}$, and $s_{i}=0$ if $0<i \leq N-1$, which is equivalent to the case of a short rectangular pulse of pulse length $[0, T / N]$. When a high range resolution is required, a large bandwidth $B$ is needed, and then, there will be a large number $M$ of range cells in a swath. This will require a large $N$. In this case, such a short pulse with length $[0, T / N]$ and power $N$ may not be easily implemented [31]. This implies that constant weights $S_{k}$ may not be a good choice for the proposed OFDM signals.

Another case is when all the weights $S_{k}$ are completely random, i.e., they are independently and identically distributed (i.i.d.). In this case, the mean power of the transmitted signal $s_{i}$ is constant for every $i$. This gives us the interesting property for an OFDM signal, namely, although its bandwidth is as large as the short pulse of length $[0, T / N]$, its mean energy is evenly spread over a much longer ( $N$ times longer) pulse duration, which makes it much easier to generate and implement in a practical system than the short pulse case.

In terms of the peak-to-average power ratio (PAPR) of transmitted signals, the former case corresponds to the worst case, i.e., the highest PAPR case that is $N$, while the later case corresponds to the best case in the mean sense, i.e., the lowest PAPR case that is 1 . After saying so, the above i.i.d. weight $S_{k}$ case is only in the statistical sense. In practice, a deterministic weight sequence $S_{k}$ is used, which can be only a pseudorandom noise (PN) sequence, and therefore, its $N$-point inverse DFT $s_{i}$ (and/or its analog waveform $s(t)$ ) may not have a constant power; in fact, its PAPR may be high (although may not be the highest) compared with the LFM radar or the random noise radar. This will be an interesting future research problem on how to deal with the high PAPR problem of OFDM signals for radar applications. Note that there have been many studies for the PAPR reduction in the communications community (see, for example, [33] and [35]). If we only consider the finite time domain signal values, i.e., the inverse discrete Fourier transform (IDFT) $s_{i}$ of the weights $S_{k}$ in (10), we can use a Zadoff-Chu sequence as $S_{k}$ that is, in fact, a discrete LFM signal, and then its IDFT $s_{i}$ has a constant module as well [37], [38]. In this case, both the weights $S_{k}$ and the discrete time domain signal values $s_{i}$ have a constant module, i.e., the discrete PAPR (the peak power over the mean power of $s_{i}$ ) is 1 .

As a remark, if one only considers the discrete transmitted signal sequence $s_{i}$, it can be, in fact, from any radar signal, such as the LFM or random noise radar signal as follows. 
Let $s^{\prime}(t)$ be any radar transmitted signal, and set $s_{i}=$ $s^{\prime}(i T / N), 0 \leq i \leq N-1$. Then, we can always find the corresponding weights $S_{k}, 0 \leq k \leq N-1$, that are just the $N$-point FFT of $s_{i}, 0 \leq i \leq N-1$. Then, the analog OFDM waveform $s(t)$ in (1) can be thought of as an interpolation of this discrete time sequence $s_{i}$. Thus, as $N$ goes large, the analog OFDM waveform $s(t)$ can approach the given radar waveform $s^{\prime}(t)$.

\section{Insufficient CP Case}

Let us consider the CP length to be $\bar{M}$ and $\bar{M}<M-1$. In this case, the length of CP is insufficient. When an insufficient $\mathrm{CP}$ is used, if OFDM pulses are transmitted consecutively without any waiting interval as in communications systems, the OFDM blocks will interfere each other due to the multipaths, which is called interblock interference (IBI). However, this will not occur in our radar application in this paper since the second OFDM pulse needs to wait for all the radar return signals of the first transmitted OFDM pulse to be received, as we have explained in Section II-A earlier. Although there is no IBI, the insufficient $\mathrm{CP}$ will cause the intercarrier interference (ICI) that leads to the IRCI as shown hereinafter. In this case, (13) can be recast as

$$
\begin{aligned}
\Delta \boldsymbol{u} & =\boldsymbol{u}-\overline{\boldsymbol{u}} \\
& =\boldsymbol{H} \boldsymbol{s}^{\prime}-\overline{\boldsymbol{H}} \boldsymbol{s}^{\prime}+\boldsymbol{w}
\end{aligned}
$$

where

$$
\overline{\boldsymbol{H}}=\left[\begin{array}{ccccccccc}
0 & \cdots & d_{M-1} & d_{M-2} & \cdots & d_{\bar{M}+1} & 0 & \cdots & 0 \\
0 & \cdots & 0 & d_{M-1} & \cdots & d_{\bar{M}+2} & 0 & \cdots & 0 \\
\vdots & \cdots & \vdots & \ddots & \ddots & \vdots & \vdots & \vdots & \vdots \\
0 & \cdots & 0 & 0 & \ddots & d_{M-1} & 0 & \cdots & 0 \\
\vdots & \cdots & \vdots & \ddots & \ddots & \vdots & \vdots & \cdots & \vdots \\
0 & \cdots & 0 & 0 & \cdots & 0 & 0 & \cdots & 0
\end{array}\right] .
$$

The $n$th element of $\Delta \boldsymbol{u}$ can be expressed as

$$
\Delta u_{n}=u_{n}-\bar{u}_{n}, n=M-1, M, \ldots, N+M-2
$$

where $u_{n}$ is the same as (11), i.e., from $\boldsymbol{H} \boldsymbol{s}^{\prime}$ and noise $\boldsymbol{w}$, and

$$
\bar{u}_{n}=\sum_{\tilde{m}=\bar{M}-M+2+n}^{M-1} d_{\tilde{m}} s_{n-\tilde{m}} .
$$

Notice that $\bar{u}_{n}=0$ for $n=2 M-\bar{M}-2, \ldots, N+M-2$. Then, the $N$-point FFT is performed on the vector $\Delta \boldsymbol{u}$

$$
U_{k}=D_{k} S_{k}-\bar{U}_{k}+W_{k}, \quad k=0,1, \ldots, N-1
$$

where

$$
\bar{U}_{k}=\frac{1}{\sqrt{N}} \sum_{n=0}^{N-1} \bar{u}_{n+M-1} \exp \left\{\frac{-j 2 \pi k n}{N}\right\} .
$$

After the $N$-point IFFT is performed on the vector $\left[\bar{U}_{0} / S_{0}^{\prime}, \bar{U}_{1} / S_{1}^{\prime}, \ldots, \bar{U}_{N-1} / S_{N-1}^{\prime}\right]^{T}$, we can obtain

$$
\hat{d}_{m}=\sqrt{N} d_{m}-\xi_{m}+\tilde{w}_{m}, \quad m=0,1, \ldots, M-1
$$



(a)

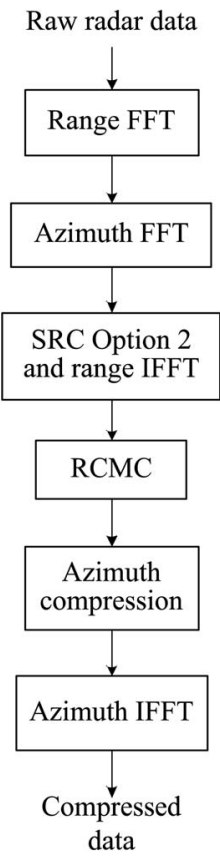

(b)

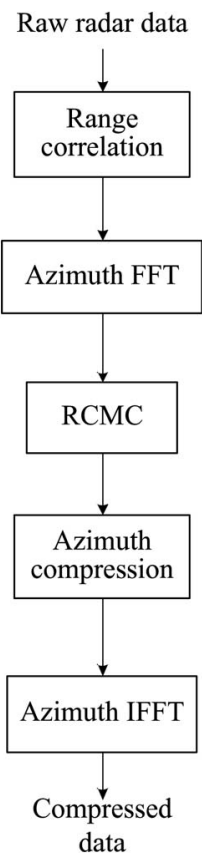

(c)
Fig. 5. Block diagram of SAR imaging processing. (a) CP-based OFDM SAR. (b) LFM SAR. (c) Random noise SAR and conventional OFDM SAR.

where

$$
\begin{aligned}
\xi_{m}=\frac{1}{N} \sum_{k=0}^{N-1} \frac{1}{S_{k}} \sum_{n=0}^{M-\bar{M}-2} \sum_{\tilde{m}=\bar{M}-M+2+n}^{M-1} d_{\tilde{m}} s_{n+M-1-\tilde{m}} \\
\times \exp \left\{\frac{-j 2 \pi k(n-m)}{N}\right\} .
\end{aligned}
$$

We remark that $\xi_{m}$ is the IRCI that resulted from the insufficient $\mathrm{CP}$, and $\xi_{m}$ is related to the reflectivities of the neighboring range cells and the transmitted signal. From the second and third summation signs in $\xi_{m}$ in (31) and (27), a smaller $\bar{M}$ leads to more range cells involved in the interference, resulting in a stronger IRCI. The performance degradation with different insufficient CP lengths of $\bar{M}$ will be shown in the simulations in Section III later.

\section{Simulations And Performance Discussions}

This section is to present some simulations and discussions for our proposed CP-based OFDM SAR imaging. The simulation stripmap SAR geometry is shown in Fig. 1. The azimuth processing is similar to the conventional stripmap SAR imaging [2] as shown in Fig. 5(a). For computational efficiency, a fixed value of $R_{c}$ located at the center of the range swath is set as the reference range cell as in [2]. Then, the range cell migration correction (RCMC) and the azimuth compression are implemented in the whole range swath using $R_{c}$. For convenience, we do not consider the noise in this section as what is commonly done in SAR image simulations. For comparison, we also consider the range Doppler algorithm using LFM and random noise signals as shown in the block diagram of Fig. 5. Since the performance of a step frequency signal SAR is similar to that of an LFM 


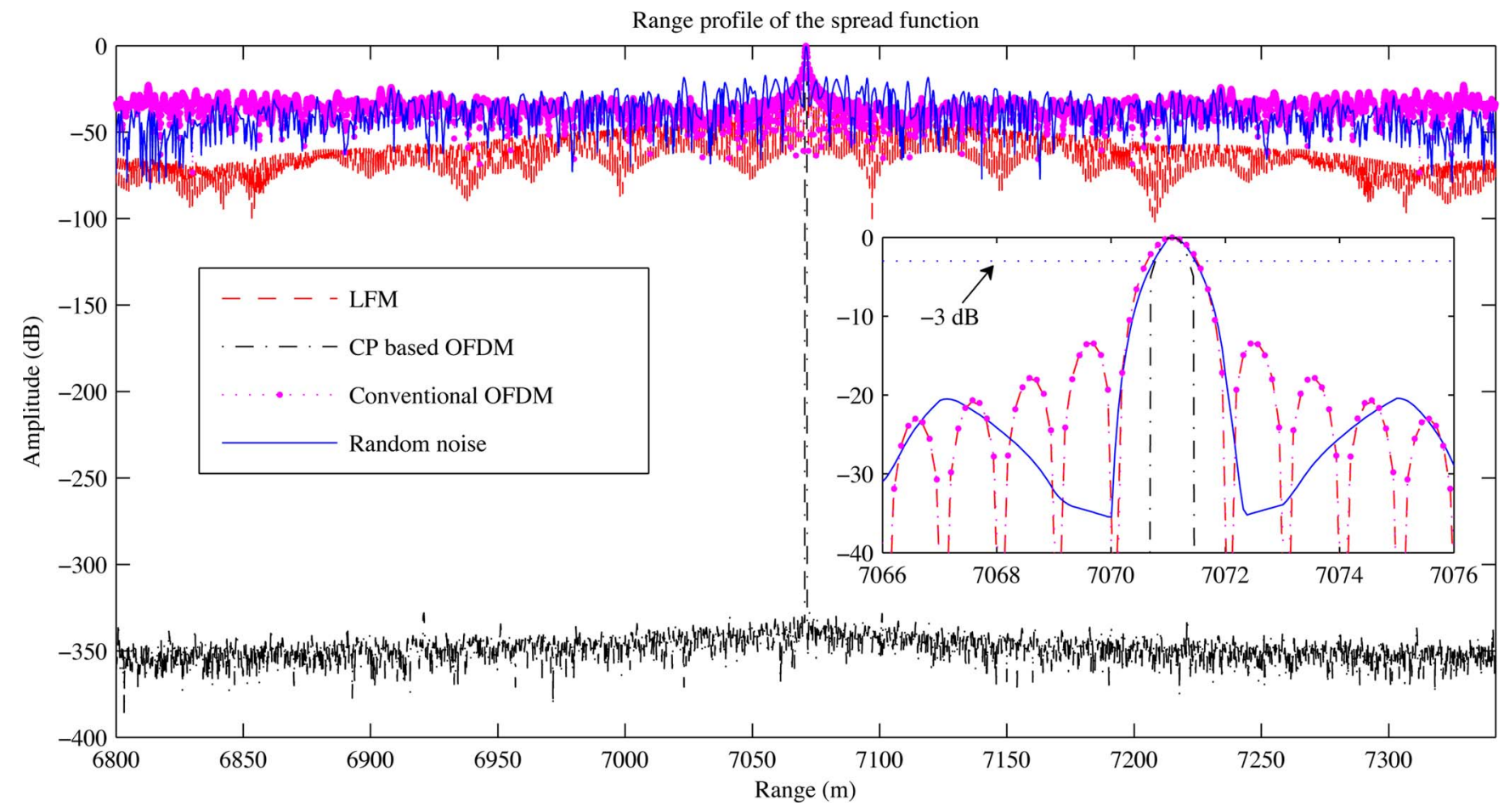

Fig. 6. Range profiles of the point spread function.

signal SAR, here, we only consider the LFM signal SAR in our comparisons. In Fig. 5(b), the secondary range compression is implemented in the range and azimuth frequency domain, the same as Option 2 in [2, Ch. 6.2]. In Fig. 5(c), the range compression of the random noise signal and the conventional OFDM signal are achieved by the correlation between the transmitted signals and the range time domain data. Notice that the difference of these three imaging methods in Fig. 5 is the range compression, while the RCMC and azimuth compression are identical.

The simulation experiments are performed with the following parameters as a typical SAR system: $\mathrm{PRF}=800 \mathrm{~Hz}$, the bandwidth is $B=150 \mathrm{MHz}$, the antenna length is $L_{a}=1 \mathrm{~m}$, the carrier frequency $f_{c}=9 \mathrm{GHz}$, the synthetic aperture time is $T_{a}=1 \mathrm{~s}$, the effective radar platform velocity is $v_{p}=150 \mathrm{~m} / \mathrm{s}$, the platform height of the antenna is $H_{p}=5 \mathrm{~km}$, the slant range swath center is $R_{c}=5 \sqrt{2} \mathrm{~km}$, the sampling frequency $f_{s}=150 \mathrm{MHz}$, and the number of range cells is $M=96$ with the center at $R_{c}$. For the convenience of FFT/IFFT computation, we set $T=(512 / 150) \mu \mathrm{s} \approx 3.41 \mu \mathrm{s}$; then, the number of subcarriers for the OFDM signal is $N=512$. The CP length is 95 that is sufficient, and the CP time duration is $T_{G I}=$ $(95 / 150) \mu \mathrm{s} \approx 0.63 \mu \mathrm{s}$. Thus, the time duration of an OFDM pulse is $T_{o}=(607 / 150) \mu \mathrm{s} \approx 4.05 \mu \mathrm{s}$. The complex weight vectors over the subcarriers of the CP-based OFDM signal and the conventional OFDM signal are set to be vectors of the binary PN sequence of values -1 and 1 . Meanwhile, for the transmission energies of the three SAR imaging methods to be the same, the time durations of an LFM pulse, a conventional OFDM pulse, and a random noise pulse are also $4.05 \mu \mathrm{s}$.

A point target is assumed to be located at the range swath center. Without considering the additive noise, the normalized range profiles of the point spread function are shown in Fig. 6,



Fig. 7. Azimuth profiles of the point spread function.

and the details around the main lobe area are shown in its zoomin image. It can be seen that the sidelobes are much lower for the CP-based OFDM signal than those of the other three signals, while the 3-dB main lobe widths of the four signals are all the same.

The normalized azimuth profiles of the point spread function of the three methods are shown in Fig. 7. The results show that the azimuth profiles of the point spread function are similar for all the four signals of LFM, CP-based OFDM, conventional OFDM, and random noise.

We then consider an extended object with the shape of a tank constructed by a few single point scatterers, and the original reflectivity profile is shown as Fig. 8(a). The results indicate that the imaging performance using the CP-based OFDM SAR is better than that of the other three signals. Specifically, the boundaries of the extended object are observed 


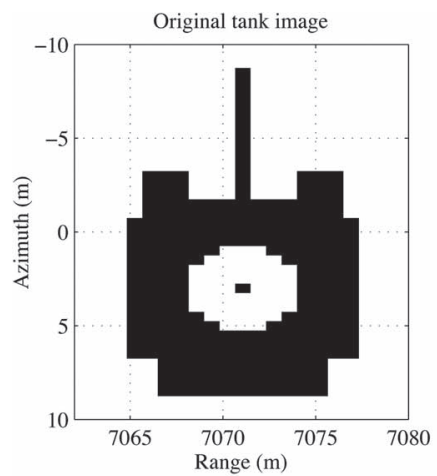

(a)

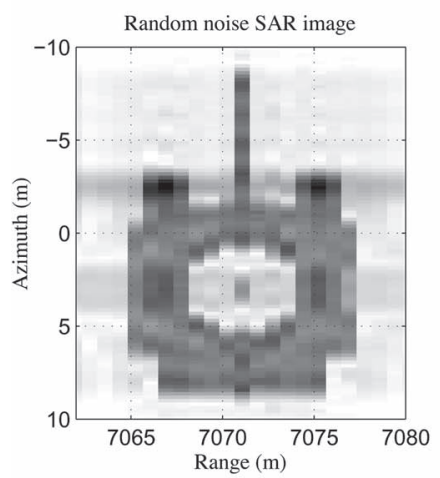

(c)



(e)

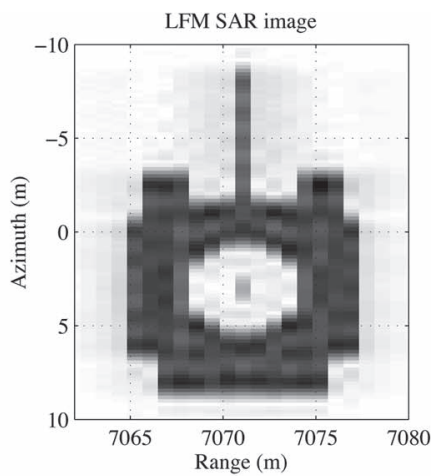

(b)

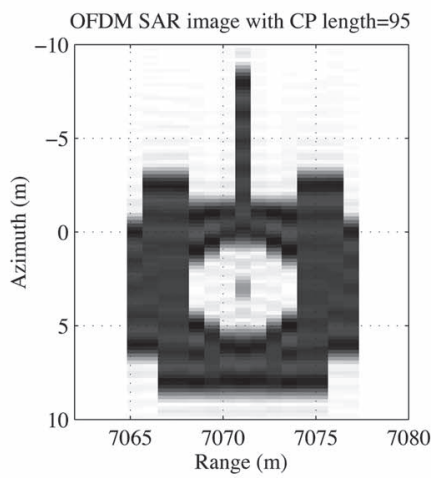

(d)

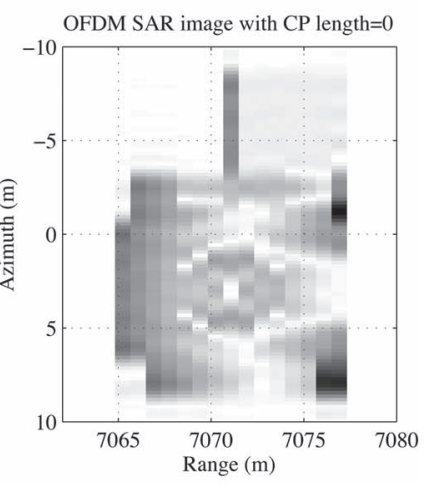

(f)
Fig. 8. Imaging results of simulated reflectivity profile for a tank. (a) Original tank. (b) LFM SAR. (c) Random noise SAR. (d) OFDM SAR with sufficient CP. (e) Conventional OFDM signal SAR. (f) CP-based OFDM SAR with $\mathrm{CP}$ length $=0$.

to be less blurred by using the CP-based OFDM SAR imaging. In Fig. 8(f), we also consider the imaging of the tank with our proposed method when the $\mathrm{CP}$ length is zero and the transmitted OFDM pulse is the same as the conventional OFDM pulse. As a remark, comparing Fig. 8(e) and (f), one may see that the SAR imaging performance degradation is significant when the CP length is zero. This is because our proposed range reconstruction method in the receiver, as mentioned in (15)-(21), is for CP-based OFDM SAR imaging and different with the traditional matched filter SAR imaging method. Thus, sufficient CP should be included in the transmitted OFDM pulse to achieve IRCI-free range reconstruction.

We next consider the importance of adding a sufficient $\mathrm{CP}$ in our proposed CP-based OFDM SAR imaging. We consider a single range line (a cross range) with $M=96$ range cells; targets are included in 18 range cells, the amplitudes are randomly generated and shown as the red circles in Fig. 9, and the RCS coefficients of the other range cells are set to be zero. The normalized imaging results are shown as the blue asterisks. The results indicate that the imaging is precise when the length of CP is 95, i.e., sufficient CP length, in Fig. 9(a), and the amplitudes of the range cells without targets are lower than $-300 \mathrm{~dB}$, which is due to the computer numerical errors. With the decrease of the CP length, the imaging performance is degraded, and the IRCI is increased. Specifically, the zero amplitude range cells become nonzero anymore, and some targets are even submerged by the IRCI as shown in Fig. 9(b) and (c). We also show the imaging results with the conventional OFDM SAR image as in Fig. 9(d). The curves in Fig. 9(d) indicate that some targets are submerged by the IRCI from other range cells. In Fig. 9, we notice that, when the CP lengths are 95 and 80 (as in Fig. 9(a) and (b), respectively), the imaging performances of our proposed method outperform that of the conventional OFDM SAR image in Fig. 9(d). However, the imaging performance with zero length $\mathrm{CP}$ is worse than that of the conventional OFDM SAR image, although they have the same transmitted OFDM waveform, which is again because our proposed range reconstruction method at the receiver is different from the conventional method. It also further indicates that a sufficient CP is important for our proposed CP-based OFDM SAR imaging.

We also consider a single range line (a cross range) with $M$ range cells, in which the RCS coefficients are set as $g_{m}=1$, $m=0, \ldots, M-1$. After the CP-based OFDM SAR imaging with different lengths of $\mathrm{CP}$, we calculate the mean square errors (MSEs) between the energy normalized imaging results and the original RCS coefficients $g_{m}$. The results are achieved from the average of 1000 independent Monte Carlo simulations and are shown in Fig. 10. The curves suggest that the performance degradation occurs when the length of CP is less than $M-1$, i.e., insufficient. The MSE is supposed to be zero when the CP length is $M-1$ that is sufficient. However, one can still observe some errors in Fig. 10, which is because errors may occur by using a fixed reference range cell $R_{c}$ in the imaging processing (i.e., RCMC and azimuth compression), and the wider swath (or a larger $M$ ) causes the larger imaging error. Thus, the MSE is slightly larger when $M$ is larger.

\section{CONCLUSION}

In this paper, by using the most important feature of OFDM signals in communications systems, namely, converting an ISI channel to multiple ISI-free subchannels, we have proposed a novel method for SAR imaging using OFDM signals with sufficient CP. The sufficient CP insertion provides an IRCI-free (high range resolution) SAR image. We first established the CP-based OFDM SAR imaging system model and then derived the CP-based OFDM SAR imaging algorithm with sufficient CP. We then showed that this algorithm has zero IRCI (or IRCI free) for each cross range. We also analyzed the influence when the CP length is insufficient. By comparing with the LFM SAR and the random noise SAR imaging methods, we then finally provided some simulations to illustrate the high-rangeresolution property of the proposed CP-based OFDM SAR 


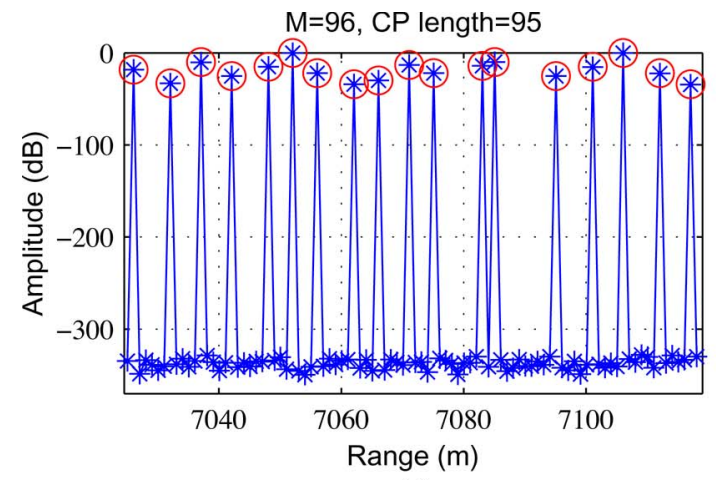

(a)



(c)

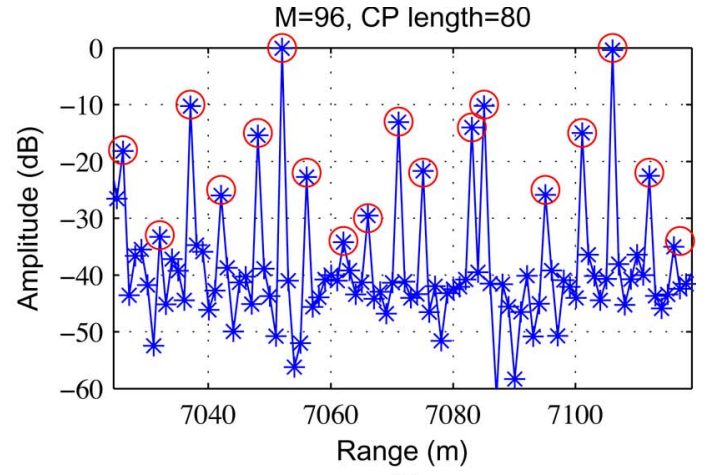

(b)

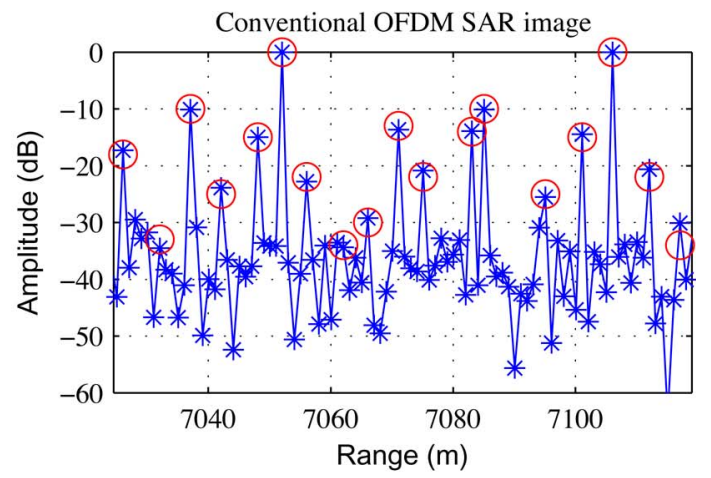

(d)

Fig. 9. Range line imaging with different CP lengths. Red circles denote the real target amplitudes, and blue asterisks denote imaging results.

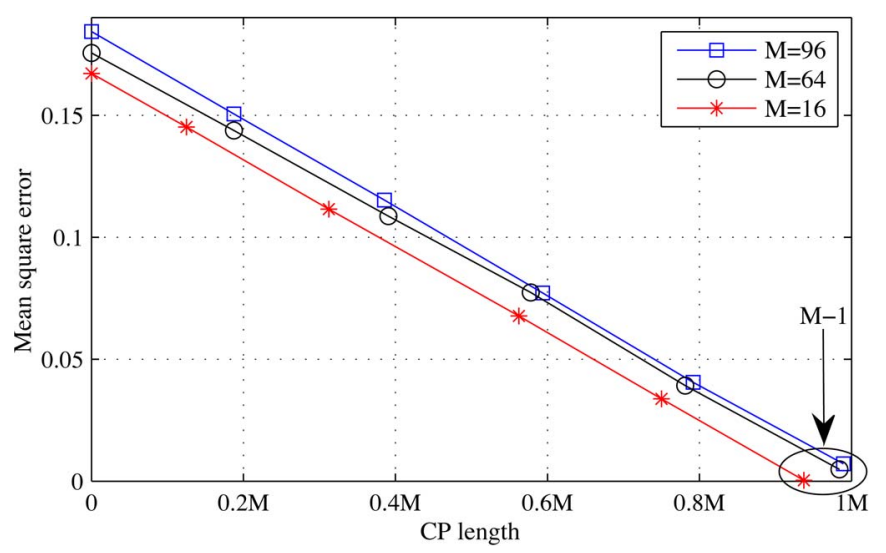

Fig. 10. MSEs for insufficient CP lengths.

imaging and also the necessity of a sufficient CP insertion in an OFDM signal. The main features of the proposed SAR imaging are highlighted as follows.

1) The sufficient $\mathrm{CP}$ length $M-1$ is determined by the number of range cells $M$ within a swath, which is directly related to the range resolution of the SAR system.

2) The optimal time duration of the OFDM pulse is $T_{o}=$ $(2 N-1) T_{s}$ with CP length $N-1$. The minimum range of the proposed CP-based OFDM SAR is the same as the maximal swath width.

3) The range sidelobes are ideally zero for the proposed CP-based OFDM SAR imaging, which can provide highrange-resolution potential for SAR systems. From our simulations, we see that the imaging performance of the CP-based OFDM SAR is better than those of the LFM SAR and the random noise SAR, which may be more sig- nificant in multiple-input multiple-output (MIMO) radar applications.

4) The imaging performance of the CP-based OFDM SAR is degraded, and the IRCI is increased when the CP length is insufficient.

Some future research works may be needed for our proposed CP-based OFDM SAR imaging systems. One of them is the high PAPR problem of the OFDM signals as what has been pointed out in Section II-C. Another problem is that OFDM signals are sensitive to an unknown Doppler shift, such as that induced from an known moving target, which may damage the orthogonality of OFDM subcarriers and result in the potential ICI. Also, how does our proposed OFDM SAR imaging method work for a distributed target model [39]? Some of these problems are under our current investigations.

\section{ACKNOWLEDGMENT}

The authors would like to thank the editor and the anonymous reviewers for their useful comments and suggestions that have improved the presentation of this paper.

\section{REFERENCES}

[1] N. C. Currie, Radar Reflectivity Measurement: Techniques and Applications. Norwood, MA, USA: Artech House, 1989.

[2] M. Soumekh, Synthetic Aperture Radar Signal Processing. New York, NY, USA: Wiley, 1999.

[3] S. R. J. Axelsson, "Analysis of random step frequency radar and comparison with experiments," IEEE Trans. Geosci. Remote Sens., vol. 45, no. 4, pp. 890-904, Apr. 2007.

[4] X. Xu and R. Narayanan, "FOPEN SAR imaging using UWB stepfrequency and random noise waveforms," IEEE Trans. Aerosp. Electron. Syst., vol. 37, no. 4, pp. 1287-1300, Oct. 2001. 
[5] D. Garmatyuk and R. Narayanan, "Ultra-wideband continuous-wave random noise arc-SAR,” IEEE Trans. Geosci. Remote Sens., vol. 40, no. 12, pp. 2543-2552, Dec. 2002.

[6] G.-S. Liu, H. Gu, W.-M. Su, H.-B. Sun, and J.-H. Zhang, "Random signal radar-A winner in both the military and civilian operating environments," IEEE Trans. Aerosp. Electron. Syst., vol. 39, no. 2, pp. 489-498, Apr. 2003.

[7] N. Levanon, "Multifrequency complementary phase-coded radar signal," Proc. Inst. Elect. Eng.—Radar, Sonar Navigat., vol. 147, no. 6, pp. 276284, Dec. 2000.

[8] G. E. A. Franken, H. Nikookar, and P. van Genderen, "Doppler tolerance of OFDM-coded radar signals," in Proc. 3rd EuRAD Conf., Manchester, U.K., 2006, pp. 108-111.

[9] D. Garmatyuk and J. Schuerger, "Conceptual design of a dual-use radar/communication system based on OFDM," in Proc. IEEE MILCOM Conf., San Diego, CA, USA, 2008, pp. 1-7.

[10] D. Garmatyuk, J. Schuerger, K. Kauffman, and S. Spalding, "Wideband OFDM system for radar and communications," in Proc. IEEE Radar Conf., Pasadena, CA, USA, 2009, pp. 1-6.

[11] C. Sturm, E. Pancera, T. Zwick, and W. Wiesbeck, "A novel approach to OFDM radar processing," in Proc. IEEE Radar Conf., Pasadena, CA, 2009, pp. 1-4.

[12] Y. L. Sit, C. Sturm, L. Reichardt, T. Zwick, and W. Wiesbeck, "The OFDM joint radar-communication system: An overview," in Proc. 3rd Int. Conf. SPACOMM, Budapest, Hungary, 2011, pp. 69-74.

[13] C. Sturm and W. Wiesbeck, "Waveform design and signal processing aspects for fusion of wireless communications and radar sensing," Proc. IEEE, vol. 99, no. 7, pp. 1236-1259, Jul. 2011.

[14] S. Sen and A. Nehorai, "Target detection in clutter using adaptive OFDM radar," IEEE Signal Process. Lett., vol. 16, no. 7, pp. 592-595, Jul. 2009.

[15] S. Sen and A. Nehorai, "Adaptive design of OFDM radar signal with improved wideband ambiguity function," IEEE Trans. Signal Process., vol. 58, no. 2, pp. 928-933, Feb. 2010.

[16] S. Sen and A. Nehorai, "OFDM MIMO radar with mutual-information waveform design for low-grazing angle tracking," IEEE Trans. Signal Process., vol. 58, no. 6, pp. 3152-3162, Jun. 2010.

[17] V. Riche, S. Meric, J. Baudais, and E. Pottier, "Optimization of OFDM SAR signals for range ambiguity suppression," in Proc. 9th EuRAD, Amsterdam, The Netherlands, 2012, pp. 278-281.

[18] V. Riche, S. Meric, E. Pottier, and J.-Y. Baudais, "OFDM signal design for range ambiguity suppression in SAR configuration," in Proc. IEEE Int. IGARSS, Munich, Germany, 2012, pp. 2156-2159.

[19] V. Riche, S. Meric, J.-Y. Baudais, and E. Pottier, "Investigations on OFDM signal for range ambiguity suppression in SAR configuration," IEEE Trans. Geosci. Remote Sens., vol. 52, no. 7, pp. 4194-4197, Jul. 2014.

[20] J.-H. Kim, M. Younis, A. Moreira, and W. Wiesbeck, "A novel OFDM chirp waveform scheme for use of multiple transmitters in SAR," IEEE Geosci. Remote Sens. Lett., vol. 10, no. 3, pp. 568-572, May 2013.

[21] D. Garmatyuk, "Simulated imaging performance of UWB SAR based on OFDM," in Proc. IEEE Int. Conf. Ultra-Wideband, Waltham, MA, USA, 2006, pp. 237-242.

[22] D. Garmatyuk and M. Brenneman, "Adaptive multicarrier OFDM SAR signal processing," IEEE Trans. Geosci. Remote Sens., vol. 49, no. 10, pp. 3780-3790, Oct. 2011.

[23] D. Garmatyuk, "Cross-range SAR reconstruction with multicarrier OFDM signals," IEEE Geosci. Remote Sens. Lett., vol. 9, no. 5, pp. 808812, Sep. 2012.

[24] C. Berger, B. Demissie, J. Heckenbach, P. Willett, and S. Zhou, "Signal processing for passive radar using OFDM waveforms," IEEE J. Sel. Topics Signal Process., vol. 4, no. 1, pp. 226-238, Feb. 2010.

[25] F. Colone, K. Woodbridge, H. Guo, D. Mason, and C. Baker, "Ambiguity function analysis of wireless LAN transmissions for passive radar," IEEE Trans. Aerosp. Electron. Syst., vol. 47, no. 1, pp. 240-264, Jan. 2011.

[26] J. R. Gutierrez Del Arroyo and J. A. Jackson, "WiMAX OFDM for passive SAR ground imaging," IEEE Trans. Aerosp. Electron. Syst., vol. 49, no. 2, pp. 945-959, Apr. 2013.

[27] P. Falcone, F. Colone, C. Bongioanni, and P. Lombardo, "Experimental results for OFDM WiFi-based passive bistatic radar," in Proc. IEEE Radar Conf., Washington, DC, USA, 2010, pp. 516-521.

[28] F. Colone, P. Falcone, and P. Lombardo, "Ambiguity function analysis of WiMAX transmissions for passive radar," in Proc. IEEE Radar Conf., Washington, DC, USA, 2010, pp. 689-694.

[29] K. Chetty, K. Woodbridge, H. Guo, and G. Smith, "Passive bistatic WiMAX radar for marine surveillance," in Proc. IEEE Radar Conf., Washington, DC, USA, 2010, pp. 188-193.
[30] Q. Wang, C. Hou, and Y. Lu, "WiMAX signal waveform analysis for passive radar application," in Proc. Int. RADAR, Bordeaux, France, 2009, pp. 1-6.

[31] M. I. Skolnik, Introduction to Radar Systems. New York, NY, USA: McGraw-Hill, 2001.

[32] X.-G. Xia, "Discrete chirp-Fourier transform and its application to chirp rate estimation," IEEE Trans. Signal Process., vol. 48, no. 11, pp. 3122 3133, Nov. 2000.

[33] R. Prasad, OFDM for Wireless Communications Systems. Boston, MA, USA: Artech House, 2004.

[34] E. Dahlman, S. Parkvall, and J. Skold, 4G: LTE/LTE-Advanced for Mobile Broadband. New York, NY, USA: Academic, 2011.

[35] A. Goldsmith, Wireless Communications. New York, NY, USA: Cambridge Univ. Press, 2005.

[36] M. Suess, B. Grafmueller, and R. Zahn, "A novel high resolution, wide swath SAR system," in Proc. IGARSS, Sydney, Australia, 2001, pp. 1013-1015.

[37] S. Beyme and C. Leung, "Efficient computation of DFT of Zadoff-Chu sequences," Electron. Lett., vol. 45, no. 9, pp. 461-463, Apr. 2009.

[38] B. Popovic, "Efficient DFT of Zadoff-Chu sequences," Electron. Lett., vol. 46, no. 7, pp. 502-503, Apr. 2010.

[39] G. Krieger, "MIMO-SAR: Opportunities and pitfalls," IEEE Trans. Geosci. Remote Sens., vol. 52, no. 5, pp. 2628-2645, May 2014.

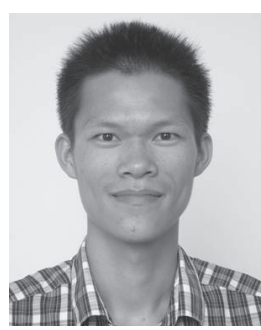

Tianxian Zhang received the B.S. degree from the University of Electronic Science and Technology of China (UESTC), Chengdu, China, in 2009. He is currently working toward the Ph.D. degree on signal and information processing at UESTC.

From October 2012, he has been a Visiting Student Researcher at the University of Delaware, Newark, DE, USA. His current research interests include radar and statistical signal processing and synthetic aperture radar systems.

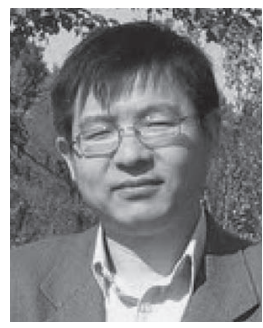

Xiang-Gen Xia (M'97-S'00-F'09) received the B.S. degree in mathematics from Nanjing Normal University, Nanjing, China, in 1983, the M.S. degree in mathematics from Nankai University, Tianjin, China, in 1986, and the Ph.D. degree in electrical engineering from the University of Southern California, Los Angeles, CA, USA, in 1992.

He was a Senior/Research Staff Member at Hughes Research Laboratories, Malibu, CA, USA, during 1995-1996. In September 1996, he joined the Department of Electrical and Computer Engineering, University of Delaware, Newark, DE, USA, where he is the Charles Black Evans Professor. His current research interests include space-time coding, MIMO and orthogonal-frequency-division-multiplexing systems, digital signal processing, and synthetic aperture radar and inverse synthetic aperture radar (ISAR) imaging. He has over 270 refereed journal articles published and accepted, is the holder of seven U.S. patents, and is the author of the book Modulated Coding for Intersymbol Interference Channels (Marcel Dekker, 2000).

Dr. Xia as the recipient of the National Science Foundation Faculty Early Career Development (CAREER) Program Award in 1997, the Office of Naval Research Young Investigator Award in 1998, and the Outstanding Overseas Young Investigator Award from the National Nature Science Foundation of China in 2001. He was also the recipient of the Outstanding Junior Faculty Award of the Engineering School of the University of Delaware in 2001. $\mathrm{He}$ is currently serving and has served as an Associate Editor for numerous international journals, including the IEEE TRANSACTIONS ON SIGNAL Processing, IEEE TransaCtions ON Wireless COMMUNiCATIONS, IEEE TRANSACTIONS ON MOBILE COMPUTING, and IEEE TRANSACTIONS on Vehicular Technology. He is the Technical Program Chair of the Signal Processing Symposium, Globecom 2007, in Washington D.C. and the General Cochair of International Conference on Acoustics, Speech and Signal Processing (ICASSP) 2005 in Philadelphia. 